\title{
Spiral Enteroscopy Assisted ERCP in Patients with Surgically Altered Anatomy: A Meta-Analysis and Systematic Review
}

\author{
Manjusha Das*, Watcoun-Nchinda Pisoh, Srinivas Puli \\ Division of Gastroenterology and Hepatology, University of Illinois College of Medicine, Peoria, Illinois, USA \\ Email address: \\ Manjusha.das@osfhealthcare.org (M. Das), Srinivas.Puli@osfhealthcare.org (W. Pisoh), \\ Watcoun-Nchinda.E.Pisoh@osfhealthcare.org (S. Puli) \\ ${ }^{*}$ Corresponding author
}

\section{To cite this article:}

Manjusha Das, Watcoun-Nchinda Pisoh, Srinivas Puli. Spiral Enteroscopy Assisted ERCP in Patients with Surgically Altered Anatomy: A Meta-Analysis and Systematic Review. Journal of Surgery. Vol. 8, No. 4, 2020, pp. 104-108. doi: 10.11648/j.js.20200804.11

Received: April 1, 2020; Accepted: May 6, 2020; Published: June 9, 2020

\begin{abstract}
Endoscopic retrograde cholangiopancreatography (ERCP) is a well-described technique for diagnosis and treatment of hepatobiliary and pancreatic disorders. Spiral enteroscopy assisted ERCP (SE-ERCP) is a well-described technique for reaching and cannulating the ampulla and performing diagnostic and therapeutic interventions. The aim of the meta-analysis is to evaluate the procedural success rate of SE-ERCP in patients with altered upper gastrointestinal anatomy. A search was conducted in Medline, Pubmed, and Ovid and extracted data into an abstraction form. Meta-analysis for success rates was analyzed by calculating pooled proportion with inverse variance. A total of 172 SE-ERCPs were included in this analysis from five relevant studies. The pooled success rate of reaching the ampulla by spiral enteroscopy was $75 \%$. SE-ERCP is a viable technique for diagnostic and therapeutic intervention of biliary disorders.
\end{abstract}

Keywords: Meta-analysis, Systematic Review, Spiral Enteroscopy, Assisted ERCP, Altered Gastrointestinal Anatomy, Roux-en-Y anatomy, Choledocholithiasis

\section{Introduction}

In patients with altered anatomy endoscopic retrograde cholangiopancreatography (ERCP) can be technically challenging and difficult to perform especially with cannulation of the biliopancreatic system. Experienced therapeutic endoscopists have increased the success rates and decreased complications rates over the past several years. As other alternative diagnostic modalities have developed, such as magnetic retrograde cholangiopancreatography (MRCP), ERCP has evolved to become predominately a therapeutic endoscopic procedure [1]. As the rates of obesity continue to rise, so have the rates of bariatric surgeries specifically Rouxen-Y gastric bypass. These particular patients are even more susceptible to choledocholithiasis and other bile duct strictures. ERCP is more difficult to perform in patients with altered anatomy.

The type of anatomy or surgical reconstruction performed will determine the accessibility of the ampulla. The route and endoscopic modality to successfully reach the ampulla depends on the length of the afferent, or biliary limb. Deviceassisted endoscopy (DAE) has been used to help aid in deep enteroscopy to reach the native ampulla. DAE includes single balloon, double-balloon (SBE or DBE), and rotational or spiral enteroscopy (SE). These assisting devices can increase ERCP success rates depending on the reconstruction type, and length of afferent limb [2] by pleating the small bowel into the overtube to stabilize the scope.

$\mathrm{SE}$ is different from SBE or DBE by rotating the overtube for gripping while pleating the small bowel onto the endoscope, and aiding the advancement of the scope into the lumen. The spiral overtube straightens the lumen and allows for more stable manipulation of the endoscope. There are currently very few studies published which studying the effectiveness of SE-ERCP in Roux-en-Y gastric bypass patients. Thus, the objective of this meta-analysis was to evaluate the procedural success rates of SE-ERCP in patients with Roux-en-Y gastric bypass anatomy. 


\section{Methods}

\subsection{Study Selection Criteria}

Studies using spiral enteroscopy assisted ERCP for Rouxen-Y gastric bypass anatomy were selected.

\subsubsection{Data Collection \& Extraction}

Only full-text published articles were included in the preliminary search. A comprehensive literature search was performed using PubMed, Ovid journals, and Medline databases. The search was performed using a combination of search terms: "Spiral enteroscopy ERCP" or "ERCP in altered anatomy". Additional criteria for inclusion were studies which noted successful bile duct cannulation rates in Roux-en-Y gastric bypass patients with device assisted ERCP. Two authors (PW and MD) independently searched and extracted data. Differences were resolved by mutual agreement. The collected data was quantified using the Cohen's $\kappa^{3}$.

\subsubsection{Quality of Studies}

Clinical trials designed with a control and treatment arm can be assessed for quality of the study. A number of criteria were used to assess the quality of a study (e.g. randomization, selection bias of the arms in the study, concealment of allocation, and blinding of outcome) $[4,5]$. Studies without a control arm were not assessed as there is no clear consensus on assessing these studies [4].

\subsection{Statistical Methods}

This meta-analysis was performed by calculating pooled proportions, such as pooled proportion of SE-ERCP which reached the ampulla, success rates of deep cannulation and successful therapeutic intervention. The pooled proportions were transformed into a quantity using Freeman-Tukey variant of the arcsine square root transformed proportion. Using inverse arcsine variance weights for the fixed effects model and DerSimonian-Laird weights for the random effects model $^{6}$ the pooled proportion was calculated as the backtransform of the weighted mean of the transformed proportions. Forrest plots were drawn to show the point estimates in each study in relation to the summary pooled estimate. The width of the point estimates in the Forrest plots indicates the assigned weight to that study. The heterogeneity among studies was tested using Cochran's $Q$ test based upon inverse variance weights [7]. If $\mathrm{p}$ value is $>0.10$, it rejects the null hypothesis that the studies are heterogeneous. The effect of publication and selection bias on the summary estimates was tested by both Harbord-Egger bias indicator [7] and Begg-Mazumdar bias indicator [8]. Also, funnel plots were constructed to evaluate potential publication bias using the standard error and diagnostic odds ratio $[8,9]$.

\section{Results}

Initial search found over 530 reference articles. Among these only 21 applied to the inclusion criteria. Ultimately, 5 studies [10-14] were relevant articles. These were selected and appraised. All 5 studies $(\mathrm{N}=172)$ met inclusion criteria and were published as full text articles. All the pooled estimates given are estimates calculated by the fixed effect model. The pooled proportion of SE-ERCP to reach the ampulla was $74.60 \%(95 \% \mathrm{CI}=67.90$ to 80.77$)$. The Forrest plot is shown in Figure 1. Duct cannulation which was indicated showed a pooled proportion of $72.96 \%(95 \%$ $\mathrm{CI}=66.14$ to 79.27$)$, shown in Figure 2 . The rate of adverse events noted was $15.11 \%(\mathrm{~N}=26)$. The most common post procedure related complication was post ERCP pancreatitis. Two perforations were observed in all the studies.

The pooled proportion of successful deep cannulation and cholangiography was $63.36 \%(95 \% \mathrm{CI}=50.34$ to 73.79$)$, shown in Figure 3. Successful therapeutic interventions pooled proportion of $58.55 \%(95 \% \mathrm{CI}=36.34$ to 79.06$)$ which is shown in Figure 4. The agreement between reviewers for the collected data gave a Cohen's $\kappa$ value of 1.0. Publication bias calculated using Harbord-Egger bias indicator gave a value of $-1.32(95 \% \mathrm{CI}=-7.76$ to $5.14, p=0.57)$. The BeggMazumdar indicator gave a Kendall's tau $b$ value of 0 $(p=0.82)$. Funnel plot for publication bias is shown in Figure 5 .

\section{Discussion}

Performing an ERCP with altered anatomy is very challenging and requires the endoscopist to consider the feasibility of reaching the ampulla as well as completing necessary diagnostic and therapeutic tasks. Success rates are typically lowest in patients with Roux-en-Y gastric bypass [13] due to the angulation and long Roux limb. The pooled success rates of reaching the ampulla by SE was high at $75 \%$. Also, the pooled success rate of deep cannulation was equally as high at $73 \%$. This indicates SE-ERCP as a successful tool to use in specifically Roux-en-Y patients to aid in not only reaching the ampulla but cannulation as well. Overall failure to reach the ampulla is more so due to procedural failure of getting into the bile duct, and not due to failure of SE-ERCP. The spiral overtube allows for better stabilization of the scope and thus advancement to the ampulla.

Kogure et al [15] described the ease of use, the control of endoscope, and quick learning curve as advantages of rotational devices compared to balloon devices. It is compatible with either of the currently available enteroscopes and does not require a separate balloon inflation system. Several previously reported studies indicate using spiral enteroscopy and successful cannulation after failed balloon enteroscopy. Lennon et al [12] suggested that the only factor associated with increased diagnostic yield was operator experience but only for balloon assisted and not SE-ERCP. However, this information was not collected systematically and may affect the overall success rates.

Adverse outcomes and procedure related complications were low. The rate of perforation was $1 \%$, and were related to needle-knife stricturoplasty, and not the spiral enteroscopy itself. Limitations of this study included the small sample 
size, as there are very few published studies investigating spiral enterosocopy alone. Moreover, factors which affect the success of reaching the ampulla the such as, patient repositioning, and experience of the endoscopist was not accounted for. Several studies also have reporting biases, which may have affected the general results of the systematic review. Therefore, making a definitive comment on risk and type of complication related to the procedure is difficult.

\section{Conclusions}

Spiral assisted ERCP can be considered a successful approach prior to surgical approach if necessary. SE assisted ERCP is a safe and viable option, and comparable to other non-surgical endoscopic techniques in Roux-en-Y gastric bypass reconstruction. Theoretically, SE-ERCP has quicker insertion with shorter procedure times, and better scope control compared to balloon assisted ERCP.

Proportion meta-analysis plot [fixed effects]

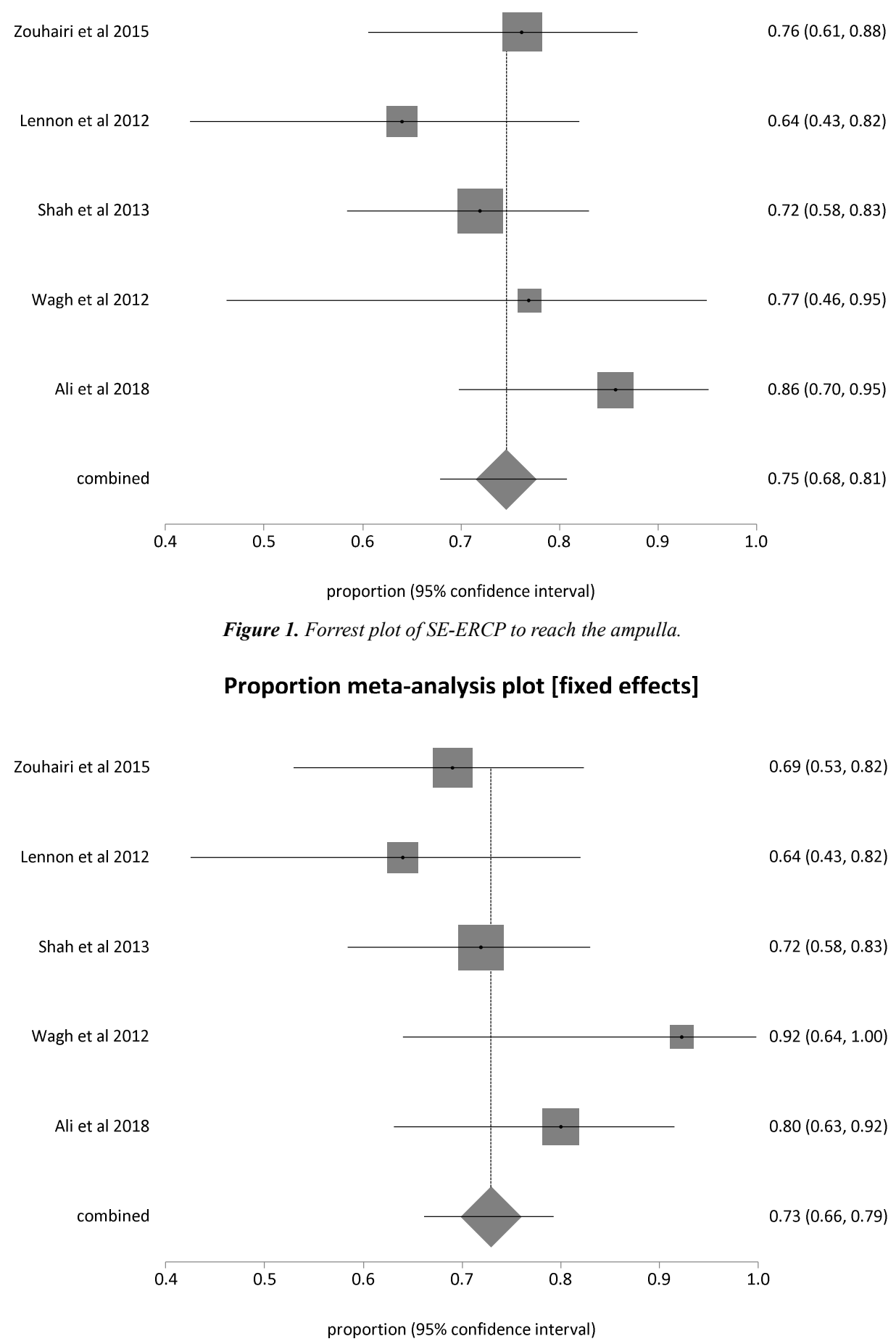

Figure 2. Forrest plot of duct cannulation which was indicated. 


\section{Proportion meta-analysis plot [fixed effects]}

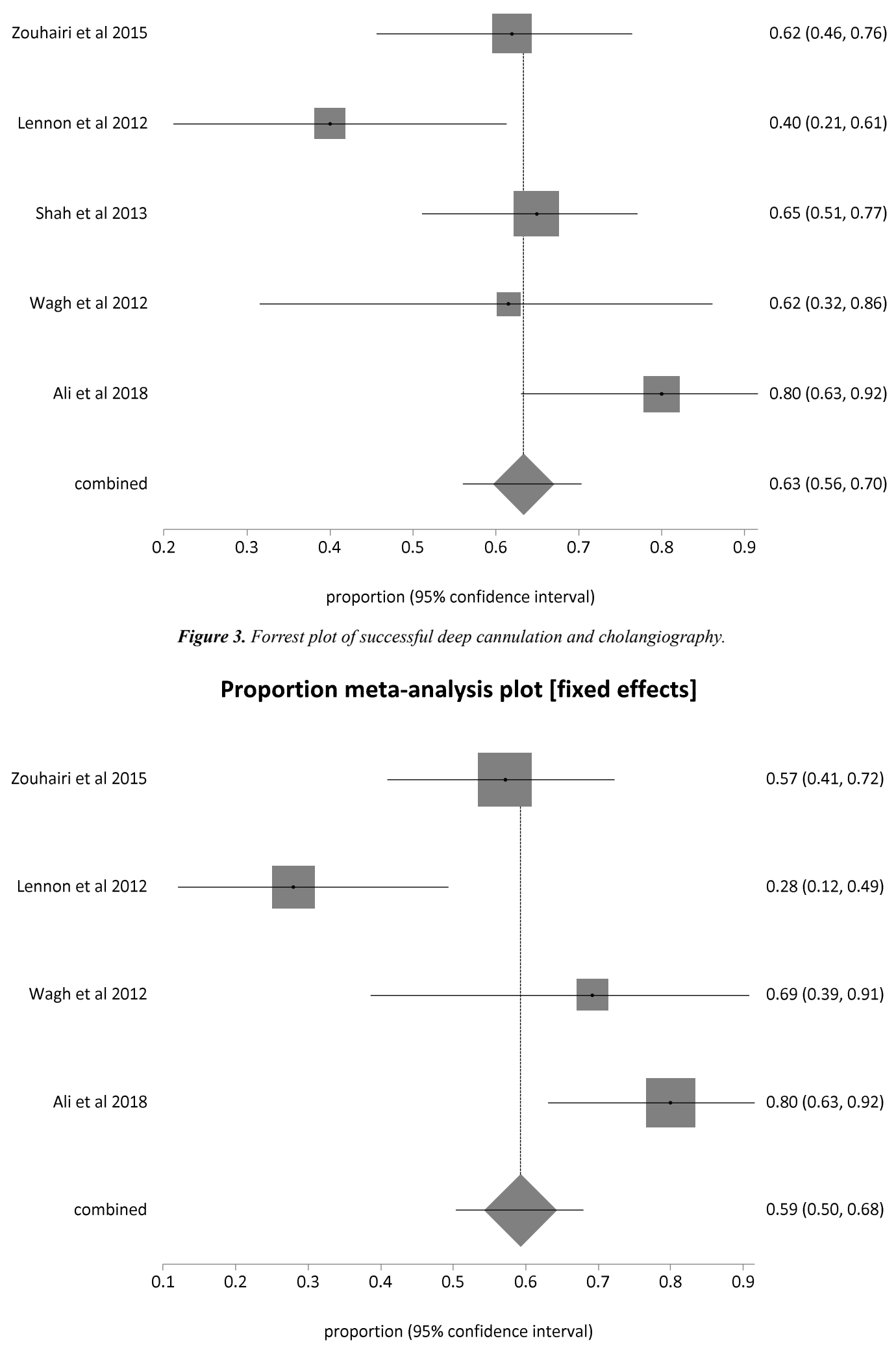

Figure 4. Forrest plot of successful therapeutic interventions. 


\section{Bias assessment plot}

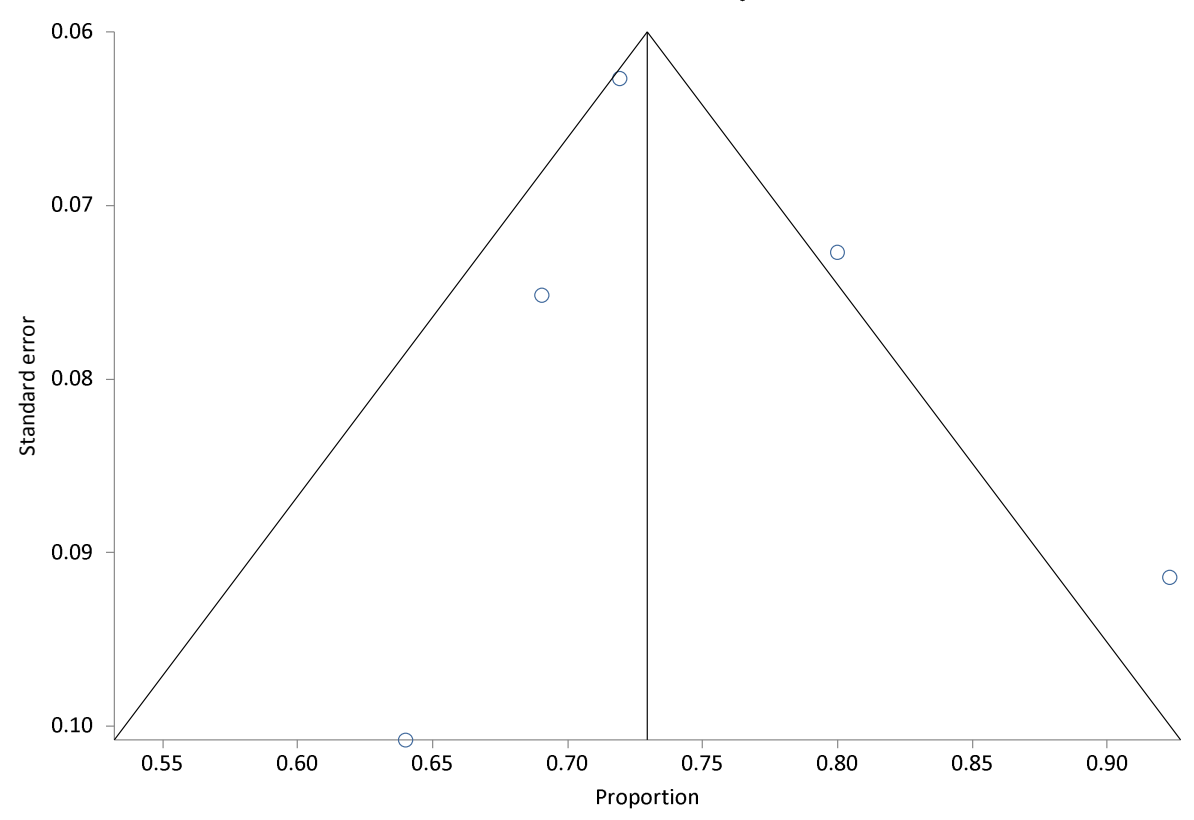

Figure 5. Funnel plot for publication bias.

\section{Conflicts of Interest and Source of Funding}

The authors declare that there is no conflict of interest regarding the publication of this paper.

\section{Acknowledgements}

No Financial support/disclosures.

\section{References}

[1] Moreels TG. Techniques for endoscopic retrograde cholangiopancreatography in altered gastrointestinal anatomy. Curr Opin Gastroenterol. 2017; 33 (5): 339-345.

[2] Krutsri C, Kida M, Yamauchi H, Iwai T, Imaizumi H, Koizumi W. Current status of endoscopic retrograde cholangiopancreatography in patients with surgically altered anatomy. World J Gastroenterol. 2019; 25 (26): 3313-3333.

[3] Brennan P, Silman A. Statistical methods for assessing observer variability in clinical measures. BMJ. 1992; 304 (6840): 1491-1494.

[4] Jadad AR, Moore RA, Carroll D, et al. Assessing the quality of reports of randomized clinical trials: is blinding necessary? Control Clin Trials. 1996; 17 (1): 1-12.

[5] Stroup DF, Berlin JA, Morton SC, et al. Meta-analysis of observational studies in epidemiology: a proposal for reporting. Meta-analysis Of Observational Studies in Epidemiology (MOOSE) group. JAMA. 2000; 283 (15): 20082012.

[6] DerSimonian R, Laird N. Meta-analysis in clinical trials. Control Clin Trials. 1986; 7 (3): 177-188.
[7] Deeks JJ. Systematic reviews in health care: Systematic reviews of evaluations of diagnostic and screening tests. BMJ. 2001; 323 (7305): 157-162.

[8] Begg CB, Mazumdar M. Operating characteristics of a rank correlation test for publication bias. Biometrics. 1994; 50 (4): 1088-1101.

[9] Sterne JA, Egger M, Smith GD. Systematic reviews in health care: Investigating and dealing with publication and other biases in meta-analysis. BMJ. 2001; 323 (7304): 101-105.

[10] Shah RJ, Smolkin M, Yen R, et al. A multicenter, U.S. experience of single-balloon, double-balloon, and rotational overtube-assisted enteroscopy ERCP in patients with surgically altered pancreaticobiliary anatomy (with video). Gastrointest Endosc. 2013; 77 (4): 593-600.

[11] Wagh MS, Draganov PV. Prospective evaluation of spiral overtube-assisted ERCP in patients with surgically altered anatomy. Gastrointest Endosc. 2012; 76 (2): 439-443.

[12] Lennon AM, Kapoor S, Khashab M, et al. Spiral assisted ERCP is equivalent to single balloon assisted ERCP in patients with Roux-en-Y anatomy. Dig Dis Sci. 2012; 57 (5): 1391-1398.

[13] Ali MF, Modayil R, Gurram KC, Brathwaite CEM, Friedel D, Stavropoulos SN. Spiral enteroscopy-assisted ERCP in bariatric-length Roux-en-Y anatomy: a large single-center series and review of the literature (with video). Gastrointest Endosc. 2018; 87 (5): 1241-1247.

[14] Zouhairi ME, Watson JB, Desai SV, et al. Rotational assisted endoscopic retrograde cholangiopancreatography in patients with reconstructive gastrointestinal surgical anatomy. World $J$ Gastrointest Endosc. 2015; 7 (3): 278-282.

[15] Kogure H, Watabe H, Yamada A, et al. Spiral enteroscopy for therapeutic ERCP in patients with surgically altered anatomy: actual technique and review of the literature. J Hepatobiliary Pancreat Sci. 2011; 18 (3): 375-379. 